\title{
Linearisation Method of DML-based Transmitters for Optical Communications Part II: Experimental Demonstration and Implementation Methods
}

\author{
N. Bamiedakis, D. G. Cunningham, Member, IEEE, and R.V. Penty, Senior Member, IEEE
}

\begin{abstract}
In this paper, we report the first experimental demonstration of the Stretched A linearisation method. The theoretical framework of the method and related simulation studies have been presented in Part I of this work. Here, we apply the method on an $850 \mathrm{~nm}$ multimode vertical-cavity surfaceemitting laser (VCSEL) specified for operation at $10 \mathrm{~Gb} / \mathrm{s}$ and demonstrate good quality optical output waveforms with little non-linear distortion at $16 \mathrm{~Gb} / \mathrm{s}$ using non-return-to-zero (NRZ) modulation. An experimental sensitivity analysis on the generation of the required modulating current components demonstrates large tolerance to parameter mismatch. In addition, it is shown that an adaptive gain scheme can improve the tolerance to the timing offset between the current components. Potential hardware implementations using either analog or digital electronics are also discussed.
\end{abstract}

Index Terms - directly-modulated lasers, optical links, nonlinearity, linearization method, optical transmitters, vertical cavity surface emitting lasers

\section{INTRODUCTION}

$\mathrm{T}$ The high data rate performance of optical links based on directly-modulated lasers (DMLs) is severely impaired by the non-linear behaviour of the laser. To address this issue, we have developed a new linearization method for optical transmitters based on DMLs, named the "Stretched A" (StrA) method. In Part I of this work, we presented its theoretical background and reported detailed simulation studies on its use in an optical link using a vertical-cavity surface-emitting laser (VCSEL) [1]. In this paper, we report the first experimental demonstration of the Stretched A method and discuss various hardware implementations.

The Stretched A method generates a current that approximates the ideal modulating current that produces an optical output waveform which is a superposition of time shifted versions of a target pulse response. For the purposes of this paper, we will call such waveforms "linear" waveforms.

Manuscript received March xx, 2021; revised May xx, 2021; accepted June xx, 2021. Date of publication December xx 2021; date of current version November xx, 2021.

N. Bamiedakis, D.G. Cunningham and R. V. Penty are with the Centre for Photonic Systems, Electrical Engineering Division, Department of Engineering, University of Cambridge, Cambridge, CB3 0FA, UK.

(e-mail: nb301@cam.ac.uk). Digital Object Identifier xxxx
Theoretically for non-return-to-zero (NRZ) modulation, as shown in Part I [1], the method efficiently corrects most of the laser non-linearity and has large tolerances.

Although the Stretched A method could be applied to any DML, the proof-of-concept experimental study reported here, as in Part I, is focussed on VCSELs. VCSEL-based links comprise the vast majority of short-reach optical links [2] and considerable effort is currently being made to improve the data rates that these can achieve [3, 4]. Using the proposed Stretched A method, good quality output optical waveforms with little non linear distortion are achieved at $16 \mathrm{~Gb} / \mathrm{s}$ using an $850 \mathrm{~nm}$ multimode VCSEL specified for $10 \mathrm{~Gb} / \mathrm{s}$ operation. In addition, a sensitivity analysis is carried out experimentally demonstrating that the method features large tolerances.

The remainder of the paper is structured as follows. Section II briefly reviews the key points of the proposed Stretched A method. Section III presents the proof-of-principle experiments while Section IV discusses the different possible implementations in practical systems. Finally, section V provides the conclusions.

\section{STRETCHED A METHOD}

The ideal modulating current $I_{b c}(t)$ that produces a linear output optical waveform can be back-calculated from the standard laser rate equations [5]. This can be expressed as the sum of four sub-currents, named $I_{A}, I_{B}, I_{C}, I_{D}$, which are related to the laser parameters, the desired photon density in the laser cavity $N_{p}(t)$ and its first and second order derivatives $N_{p}^{\prime}=\frac{d N_{p}(t)}{d t}$ and $N_{p}^{\prime \prime}=\frac{d^{2} N_{p}(t)}{d t^{2}}$ :

$$
I_{b c}(t)=I_{A}(t)+I_{B}(t)+I_{c}(t)+I_{D}
$$

This method to obtain the modulating current is called here the "ABCD method" and the exact expressions that give each subcurrent are presented in Part I [1]. $I_{D}$ groups the constant terms and is found to be the laser threshold current, $I_{C}$ includes the terms linearly proportional to the photon density $N_{p}, I_{B}$ comprises the terms proportional to the first order derivative of the photon density $N_{p}^{\prime}$ while $I_{A}$ includes the non-linear terms and terms involving the second order derivative of the photon density $N_{p}^{\prime \prime}$. Assuming that $I_{C}$ is a linear current waveform, the $I_{A}$ and $I_{B}$ sub-currents are those required to 
mitigate the laser non-linearity. However, their expressions include terms which cannot be easily generated in practice. As a result, in Part I of this work [1], we proposed a new linearization method which we named the "Stretched A" method, and which generates an approximation of the ideal back-calculated current $I_{b c}(t)$. This is done by simplifying the $I_{A}$ and $I_{B}$ expressions in three steps: by (i) omitting the least significant terms, (ii) replacing the $1 / N_{p}$ term with the inverse of the average photon density $\overline{N_{p}}$ and (iii) time shifting and/or amplitude scaling the resulting sub-currents. The details of the method have been presented in Part I [1]. The total approximate modulating current $\widetilde{I_{b c}}$ can be written in the form:

$$
\widetilde{I_{b c}}(t)=I_{A}^{s t}(t)+\widetilde{I_{B}}(t)+I_{c}(t)+I_{D}
$$

where $I_{A}^{s t}$ and $\widetilde{I_{B}}$ represent the approximations for the $I_{A}$ and $I_{B}$ sub-currents. These are related to the linear sub-current $I_{C}$ by the following expressions:

$$
\begin{gathered}
\widetilde{I_{B}}(t)=b \frac{d I_{C}(t)}{d t} \\
I_{A}^{s t}(t)=\gamma \cdot \widetilde{I}_{A}(t+d t)
\end{gathered}
$$

with

$$
\widetilde{I_{A}}(t)=a \cdot b \frac{d^{2} I_{C}(t)}{d t^{2}}
$$

where $a$ and $b$ are scaling factors that depend on the laser parameters and operating point and can be found analytically if these are known (either by design or measurements) using the equations provided in Part I [1]. Otherwise, an adaptive method could be applied. The amplitude factor $\gamma$ and timing offset $d t$ for the $I_{A}^{s t}$ component can be found adaptively using starting values of $\gamma=1$ and $d t=0$ UI. Through simulation studies, it was shown in Part I that the generation of the $I_{A}^{s t}$ and $\widetilde{I_{B}}$ component has relatively large tolerances making this scheme viable for real-world systems.

\section{PROOF-OF-PRINCIPLE DEMONSTRATION}

\section{A. Methodology and experimental setup}

For the proof-of-principle demonstration of the Stretched A method, a commercially-available $10 \mathrm{~Gb} / \mathrm{s} 850 \mathrm{~nm}$ multimode VCSEL is employed [6]. The lower data rates used in the experimental demonstration presented here in comparison with those presented in the simulation studies in Part I (50 $\mathrm{Gb} / \mathrm{s}$ ) is due to the capabilities of the equipment available.

The VCSEL is mounted onto an SMA connector and wire bonded. Its light-current characteristic and small signal frequency response are measured and shown in Fig. 1 and Fig. 2 respectively. The small signal $3 \mathrm{~dB}$ bandwidth of the device is found to be $\sim 7 \mathrm{GHz}$ for a $\sim 5 \mathrm{~mA}$ bias current which is at the middle of the linear region of the device operation.

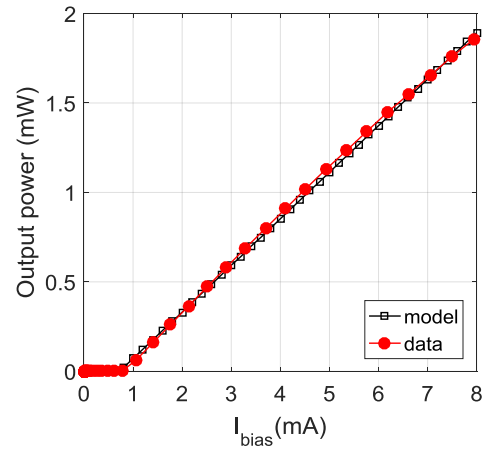

Fig. 1. Measured output optical power (coupled into a $50 \mu \mathrm{m}$ MMF via a pair of microscope objectives) as a function of the bias current. The respective values obtained with the rate equation model are also shown for comparison.

The exact device parameters are unknown but these are estimated using the measured small-signal frequency response of the device at different bias currents and the threshold current. The measured frequency response is fitted with a three-pole transfer function at each bias current $[7,8]$. This comprises a $2^{\text {nd }}$ order term $H_{\text {int }}(f)$ which describes the intrinsic dynamic behaviour of the laser, and a $1^{\text {st }}$ order term $H_{p}(f)$ which describes the combined response of the device parasitics and parasitics due to the connectorisation and mounting scheme:

$$
H(f)=|\underbrace{\frac{A \cdot f_{r}^{2}}{f_{r}^{2}-f^{2}+j\left(\frac{f}{2 \pi}\right) \gamma_{v}}}_{H_{\text {int }}(f)} \cdot \underbrace{\frac{1}{1+j\left(\frac{f}{f_{p}}\right)}}_{H_{p}(f)}|^{2}
$$

Here $A$ is a constant, $f_{r}$ and $\gamma_{v}$ describe the VCSEL resonance frequency and damping factor respectively, and $f_{p}$ is the parasitic pole. The variation of the $f_{r}$ and $\gamma_{v}$ parameters with bias current is used to estimate the intrinsic laser parameters $[7,9,10]$ after making some assumptions about the unknown laser parameters. The estimated parameters are input to the rate equation model and the simulated L-I and small signal response are obtained. These are shown in Fig. 1 and Fig. 2 for comparison with the measured data. In addition, Fig. 2(a) shows the measured, fitted and simulated small signal response at three different bias currents, Fig. 2(b) plots the laser $3 \mathrm{~dB}$ frequency as a function of bias current obtained through the measurements and simulation. The estimated $3 \mathrm{~dB}$ frequencies for the intrinsic laser operation and parasitics are also shown in Fig. 2(b). The plots demonstrate a reasonably good fit between the measured and simulated values. The differences observed at the low frequency range in Fig. 2(a) can be attributed to the parasitics induced by the wiring bonding and device mounting scheme on the SMA connector. 
(a)

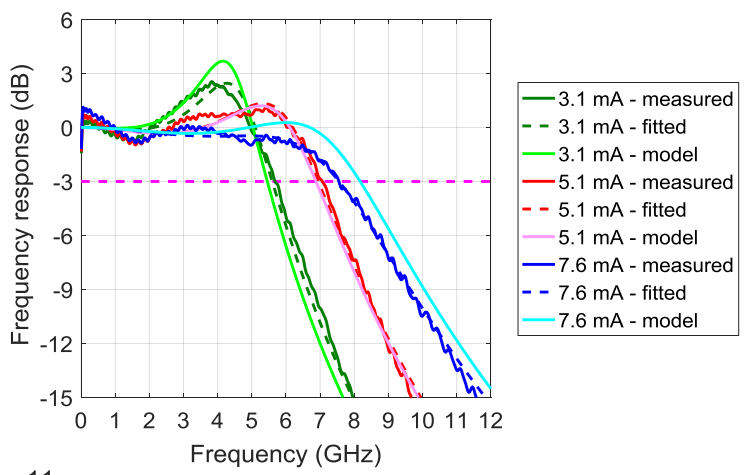

(b)

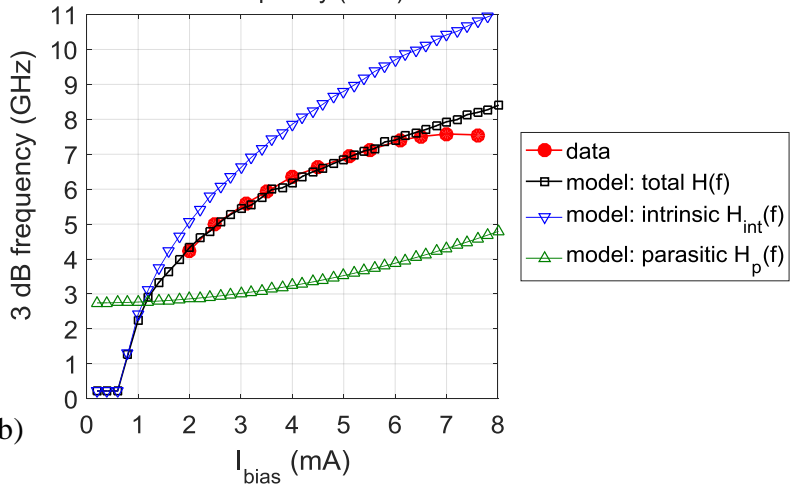

Fig. 2. (a) Measured (solid dark lines) and fitted (dashed lines) VCSEL frequency response for three bias currents. The bright solid lines show the obtained frequency response from the rate equation model using the estimated device parameters at the same three currents. (b) Measured and simulated $3 \mathrm{~dB}$ frequency for the VCSEL as a function of the bias current. The respective values for the intrinsic laser operation and device parasitic are also shown.

The high-speed performance of the VCSEL is studied experimentally and via simulations when three different modulating currents are applied: (i) the conventional NRZ modulation without any correction for non-linearity, (ii) the ideal back-calculated case (ABCD method) and (iii) the approximate current obtained with the Stretched A method. A $2^{7}-1$ pseudorandom binary sequence (PRBS-7) is used as the data pattern for the initial data transmission tests while and the "IEEE" pulse response (see Part I, section III.A [1]) with a rise time $T_{c}$ of $0.75 \times T$ is set as the desired pulse response.

The modulating current waveform for cases (ii) and (iii) is obtained using the equations presented in Part I [1]). Once these are derived, linear pre-distortion is applied onto them to correct for the parasitic transfer function and ensure that the shape of modulating current reaching the laser is as close as possible the desired one. The parasitic pole is found to be at $\sim 3.5 \mathrm{GHz}$ at $5 \mathrm{~mA}$ bias [Fig. 2(b)].

The modulating waveforms are calculated and appropriately sampled offline and loaded into an arbitrary waveform generator (AWG, Tektronix 70001A) with $14 \mathrm{GHz}$ analog bandwidth and a maximum output sampling rate of 50 GS/s. The successful implementation of the ABCD and Stretched A methods relies primarily on the successful generation of the high-frequency sub-currents $I_{A}$ and $\widetilde{I_{A}}$ to correct the non-linearity of the output waveforms. Based on the simulation studies presented in Part I [1], their successful implementation should enable data transmission at a symbol rate which is roughly double the $3 \mathrm{~dB}$ intrinsic laser

bandwidth. For the VCSEL used here, the rate equation model estimates this to be $9 \mathrm{GHz}$ at the bias current of $5.3 \mathrm{~mA}$ [Fig. 2(b)]. This suggests that data transmission of $\sim 18 \mathrm{~Gb} / \mathrm{s}$ should be possible with this device, provided that the frequency response of the parasitics can be effectively equalized. However, the generation of high-quality modulating waveforms at $18 \mathrm{~Gb} / \mathrm{s}$ has proven to be challenging due to the capabilities of the available AWG. As a result, the main data transmission experiments reported here are carried out at 16 $\mathrm{Gb} / \mathrm{s}$ which allows the generation of the modulating current with an oversampling ratio (OSR) of 3 (48 GS/s). However, some data transmission tests at $18 \mathrm{~Gb} / \mathrm{s}$ have also been carried out (OSR of 2, $36 \mathrm{GS} / \mathrm{s})$ and are reported here.

The experimental setup used in the data transmission tests is illustrated in Fig. 3. The VCSEL is biased at $\sim 5.3 \mathrm{~mA}$. The modulating current is generated by the AWG, amplified appropriately with a $40 \mathrm{GHz} \mathrm{RF}$ amplifier and fed to the VCSEL via a high-bandwidth bias tee. A pair of microscope objectives $(16 \times$ and $10 x)$ is used to couple the emitted light into a $50 \mu \mathrm{m}$ MMF patchcord. A multimode variable optical attenuator (MM VOA) is used to control the power level at the receiver. The optical sampling module (80C15) with a $32 \mathrm{GHz}$ bandwidth of an oscilloscope (Tektronix DSA8300) is used as the optical receiver for the data transmission tests. The received optical power is set to $-1.5 \mathrm{dBm}$ for all data transmission tests while the peak-to-peak amplitude of the AWG is appropriately adjusted for each test to achieve an extinction ratio for the output waveform of $\sim 5.2 \mathrm{~dB}$ for all three modulation methods. The eye diagram and the averaged trace of the received waveform are saved and processed offline. The offline processing includes the application of an 11-tap FFE at the receiver to mitigate linear distortions and the estimation of the metrics of the eye diagram: width $w$ and height $h$. The effective eye diagram area $S=w \times h$ obtained after the application of the FFE is used to assess the quality of the received waveforms obtained with the $\mathrm{ABCD}$ and Stretched A methods.

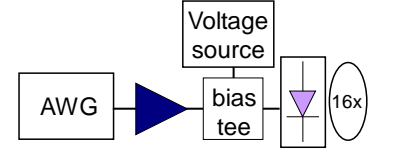

RF amplifier $850 \mathrm{~nm}$ VCSEL

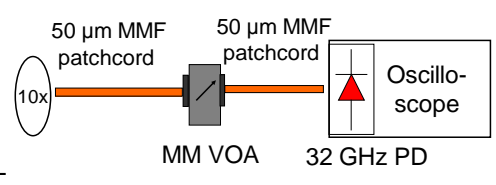

Fig. 3. Experimental setup for the data transmission experiments.

\section{B. Eye diagrams}

Fig. 4(a) to 4(c) show the recorded eye diagrams for NRZ PRBS-7 transmission at $16 \mathrm{~Gb} / \mathrm{s}$ when the conventional NRZ modulation and the $\mathrm{ABCD}$ and Stretched $\mathrm{A}$ methods are employed. It can be clearly noticed that the use of the ABCD and Stretched A methods greatly improves the linearity of the output optical waveform yielding eye diagrams with reduced level thickness, overshoot, jitter and skew.

Measurements of the noise (standard deviation of the optical power on the recorded traces) are carried out on all three output waveforms. It is found that for the same optical power, similar standard deviation values are obtained from all three output waveforms. This indicates that the proposed modulation method does not severely affect the noise levels in the output waveforms. 

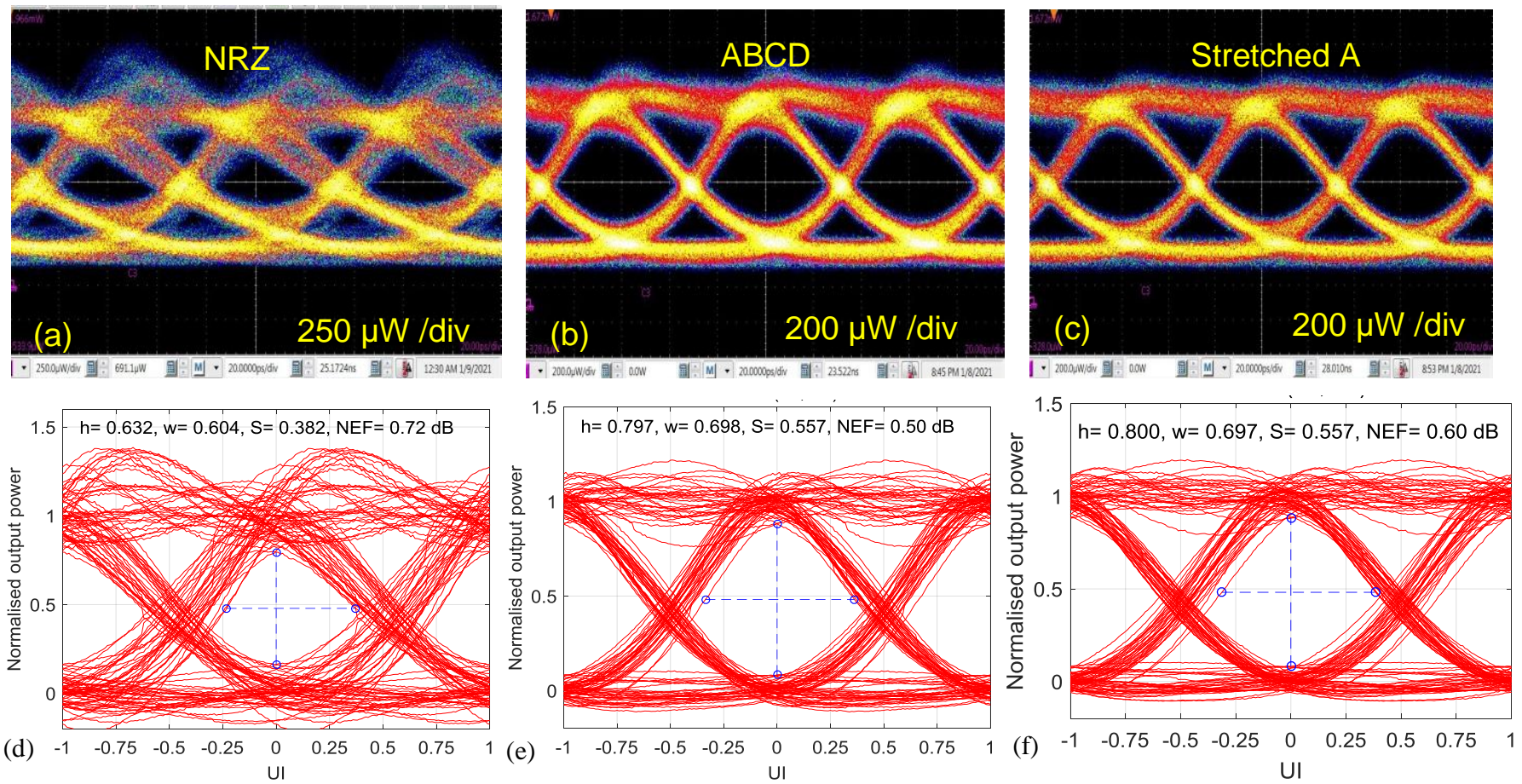

Fig. 4. Received eye diagrams at $16 \mathrm{~Gb} / \mathrm{s}$ (a-c) before and (d-f) after offline equalization for (a and d) conventional NRZ modulation, (b and e) the ABCD and (c and f) Stretched A methods. (For (a-c): time scale: 20 ps/div, NEF: noise enhancement factor).

The respective noise-free eye diagrams of the averaged optical waveform after equalization with the 11-tap FFE at the receiver are shown in Fig. 4(d)-4(f) with their eye metrics noted on the figures [width $w$, height $h$, effective area $S$ and noise enhancement factor (NEF)]. The equalized eye diagrams obtained for the ABCD and Stretched A methods are of much higher quality than that obtained with the conventional NRZ modulation. They exhibit significantly reduced jitter, level thickness and overshoot and increased effective area $S$. In addition, it can be clearly seen that the Stretched A method yields a very similar eye diagram with nearly identical eye metrics as the ABCD method. This demonstrates that the generated current is a good approximation of the ideal backcalculated current. It must be noted however, that some residual non-linearity can be observed in the equalized eye diagrams. This can be attributed to (i) the quality of the electrical signal generated by the AWG and (ii) the fact that the derivation of the modulating current waveforms is based on the crude estimation of the laser parameters described in the previous section. It is expected that better knowledge of the device physical parameters and the use of a signal generator with better characteristics will further improve the overall quality (and linearity) of the output optical waveforms.

To assess the quality of the electrical signal generated by the AWG, its electrical output waveform is recorded for conventional NRZ modulation at $16 \mathrm{~Gb} / \mathrm{s}$ after waveform averaging. Fig. 5 shows the obtained noise-free eye diagrams before and after equalization at the receiver with the (offline) 11-tap FFE equalizer with the relevant metrics noted. The eye diagrams exhibit significant jitter and level thickness with an effective area $S$ of $\sim 0.8$ after equalization. In contrast, in the simulations, the electrical modulating waveform is assumed to be ideal (zero jitter, zero level thickness and $S=1$ ).

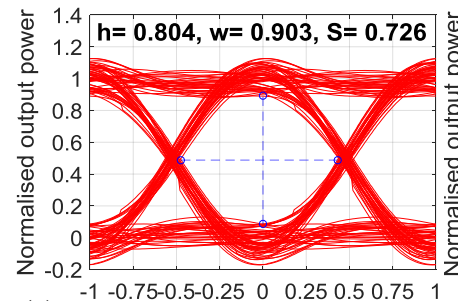

(a)

$\mathrm{UI}$

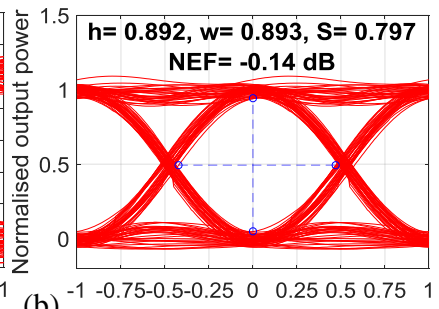

$\mathrm{UI}$
Fig. 5. Eye diagrams of the electrical output of the AWG (modulating electrical waveform) for conventional NRZ modulation at $16 \mathrm{~Gb} / \mathrm{s}$ (a) before and (b) after offline equalization with the 11-tap FFE.

The quality of the output optical waveforms for the transmission of a longer PRBS pattern, here PRBS-11, is also assessed. The eye diagrams before and after equalization of the optical waveforms generated with the three modulation currents are shown in Fig. 6. Similar observations as for the shorter PRBS-7 can be made. The ABCD and Stretched A method produce similar eye diagrams which are significantly better that those obtained with the NRZ modulation. The respective noise-free equalized diagrams confirm this with similar metrics recorded for the ABCD and Stretched A methods.

Data transmission tests are also carried out at $18 \mathrm{~Gb} / \mathrm{s}(36$ GS/s, OSR of 2) and the eye diagrams obtained for a PRBS-7 bit pattern for each modulation method are shown in Fig. 7. It can be clearly seen again that the ABCD and Stretched A methods provide similar quality eye diagrams which are much better than that for conventional NRZ modulation. However, some eye closure and skew is noticed which shows the nonlinearity has not been fully corrected. As discussed previously, this is mainly due to the quality of the electrical modulating 
waveform generated with the equipment available. As a result, the rest of the paper is focussed on $16 \mathrm{~Gb} / \mathrm{s}$ data transmission.
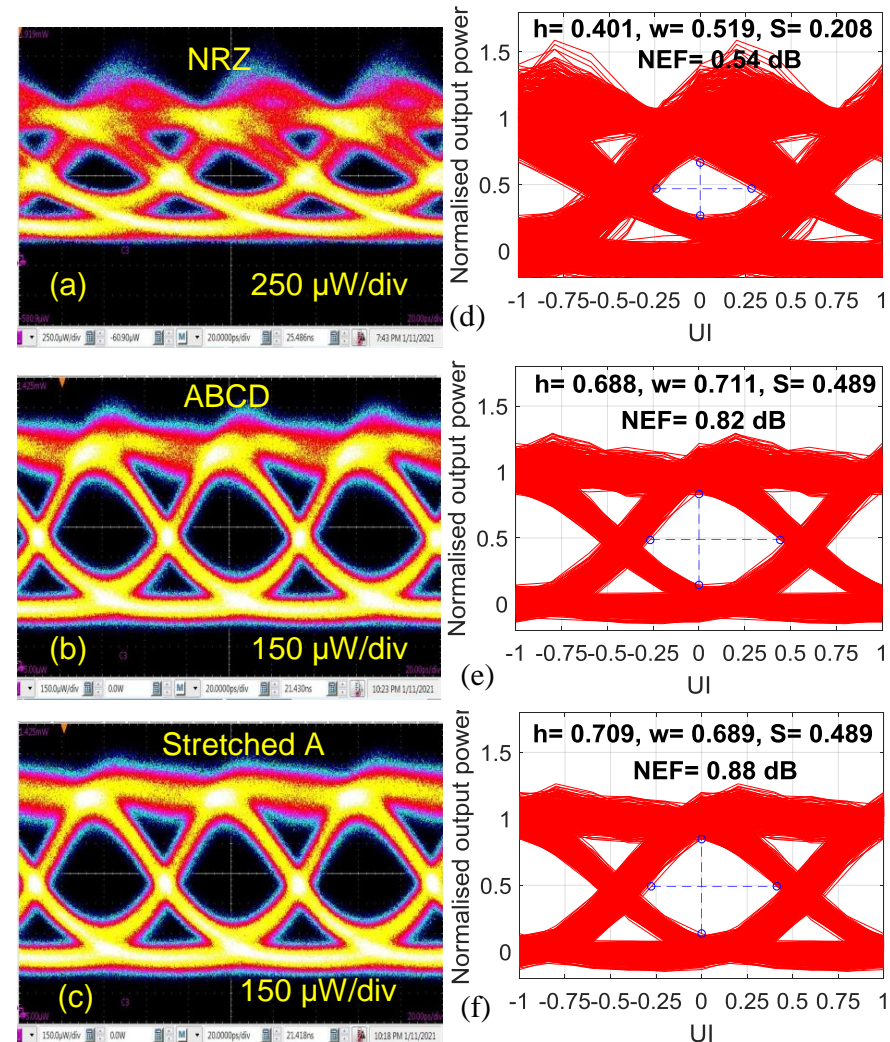

Fig. 6. Eye diagrams for the transmission of PRBS-11 at $16 \mathrm{~Gb} / \mathrm{s}$ (ac) before and after ( $\mathrm{d}-\mathrm{f})$ offline equalization for the three modulating currents studied: ( $a$ and d) conventional NRZ, (b and e) ABCD method and (c and f) Stretched A method. Time scale: 20 ps/div.

(a)

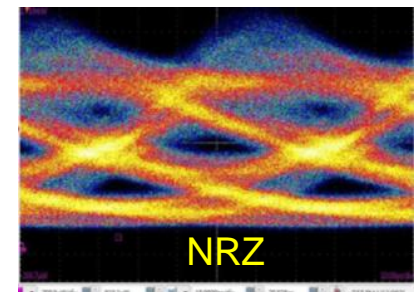

(b)
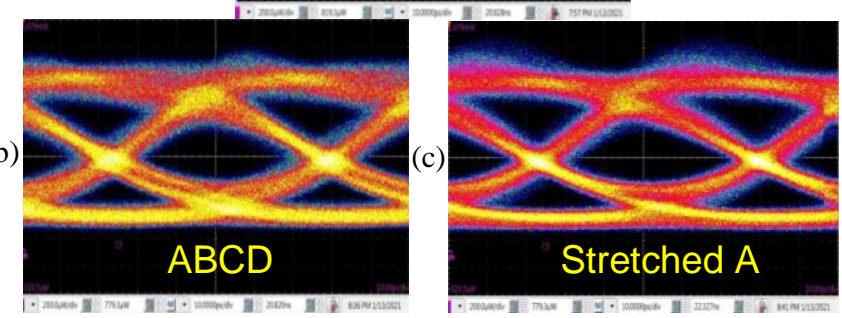

Fig. 7. Received eye diagrams at $18 \mathrm{~Gb} / \mathrm{s}$ for (a) conventional NRZ modulation, (b) the ABCD and (c) Stretched A methods. Vertical scale: $200 \mu \mathrm{W} / \mathrm{div}$, horizontal scale: $10 \mathrm{ps} / \mathrm{div}$.

\section{SENSITIVITy ANALYSIS FOR THE StRETCHED A METHOD}

A sensitivity study similar to the one presented in section IV.A of Part I [1] on the implementation of the Stretched A method is carried out experimentally on the $I_{A}^{s t}$ and $\widetilde{I_{B}}$ components for $16 \mathrm{~Gb} / \mathrm{s}$ data transmission.

\section{A. Tolerances for the $I_{A}^{s t}$ and $\widetilde{I_{B}}$ sub-current generation}

The amplitude and timing of the $I_{A}^{s t}$ component are varied according to:

$$
I_{A}^{s t}(t)=\gamma \cdot \widetilde{I_{A}}(t+d t)
$$

The $\gamma$ parameter represents the amplitude scaling while the $d t$ offset represents duty cycle distortion (DCD) of the modulating NRZ waveform as illustrated in Fig. 1 of Part I [1]. This offset due to DCD generates a reverse symmetric delay for the positive $(0 \rightarrow 1)$ and negative $(1 \rightarrow 0)$ transitions for the $I_{A}^{s t}$ sub-current: a positive $d t$ value generates a time advance for positive transitions $(0 \rightarrow 1)$ and a time delay for negative ones $(1 \rightarrow 0)$. This is illustrated in the implementation section that follows (see Fig. 12 and Fig. 13).

For each set of parameters, the average trace of the output optical waveform is recorded and the 11-tap FFE is applied offline to equalize any linear distortions in the waveform. The effective area $S$ of the eye diagram obtained after equalization is calculated and used to compare the quality of the output waveform. The maximum $S$ is found to be 0.59 for $\gamma=1.05$ and $d t=0.10$ UI. The obtained $S$ values are normalised to this maximum value and are plotted in Fig. 8(a) to obtain the tolerances of the method for the generation of the $I_{A}^{s t}$ component. The respective plot obtained through simulation using the laser rate equations is shown in Fig. 8(b) for comparison. Good agreement is achieved between the experimental and simulation results. However, it must be noted that the actual values of the eye diagram area $S$ obtained through the simulation are much larger as an ideal modulating current has been assumed as the model input. Fig. 8 also indicates that good quality eye diagrams can be obtained for a wide range of values for the scaling factor $\gamma$ and DCD timing offset $d t$ demonstrating the potential of the Stretched A method for real-world implementation.

A similar sensitivity analysis is carried out experimentally for the $\widetilde{I_{B}}$ component of the Stretched A method.

$$
\widetilde{I_{B}^{\text {off }}}(t)=\delta \cdot \widetilde{I_{B}}\left(t+d t_{B}\right)
$$

The $\delta$ parameter represents the amplitude scaling while the offset $d t_{B}$ the misalignment from the ideal transition position. The parameters of the $I_{A}^{s t}$ component are chosen as $\gamma=1.05$ and $d t=0.10 \mathrm{UI}$ as these were found to provide the best performance. Fig. 9 shows the normalized eye diagram area after equalization as a function of the values of $\delta$ and $d t_{B}$. As predicted by the simulations (section IV.A in Part I [1]), the best performance is achieved when the $\widetilde{I_{B}}$ pulse is perfectly aligned with each transition edge: $\delta=1$ and $d t_{B}=0.0 \mathrm{UI}$. In addition, Fig. 9 clearly demonstrates large tolerances of the Stretched A method for both the amplitude and timing offset of the $\widetilde{I_{B}}$ component. 

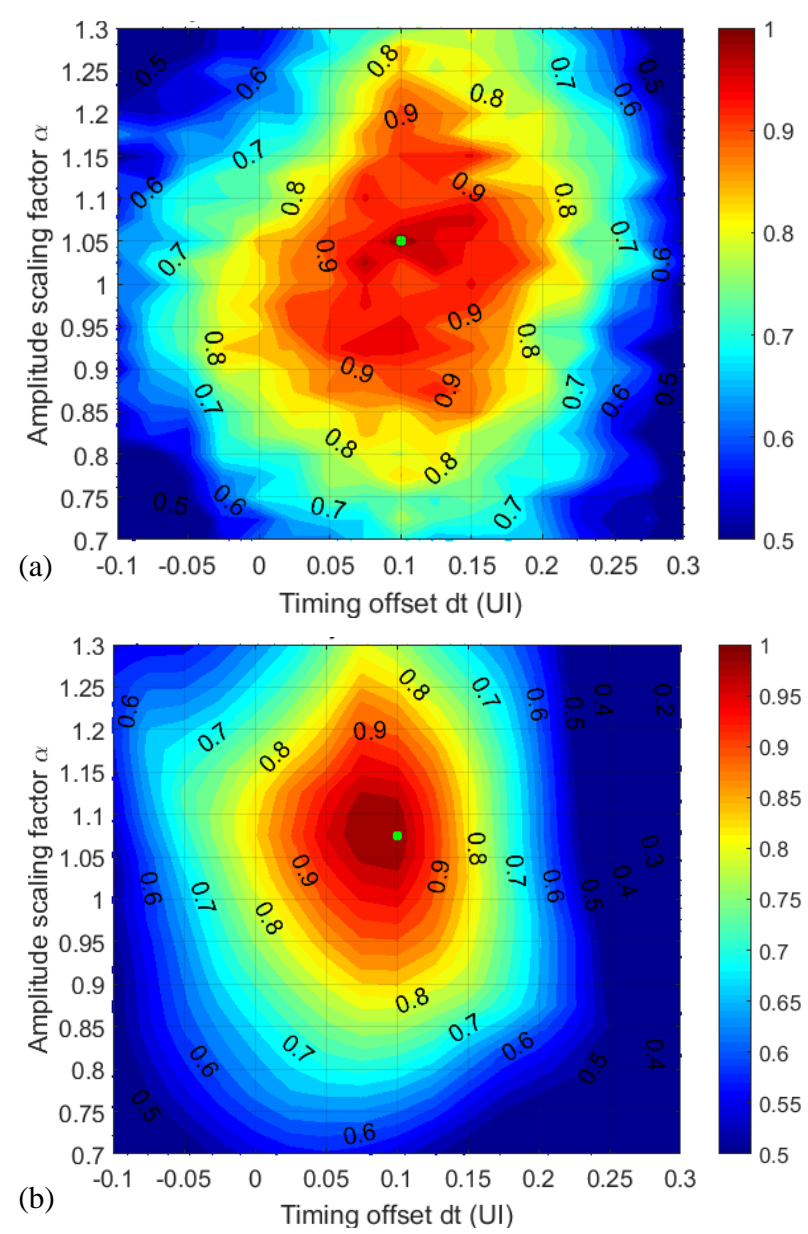

Fig. 8. Normalised eye diagram effective area $S$ after offline equalization as a function of the scaling factor $\gamma$ and DCD timing offset $d t$ of the $I_{A}^{s t}$ component of the Stretched A method for $16 \mathrm{~Gb} / \mathrm{s}$ transmission obtained (a) experimentally and (b) through simulations. The green dot in the plots indicates the location of the maximum value recorded: (a) experiments: $\max S=0.59$ for $\gamma=1.05$ and $d t$ $=0.10$ UI, (b) simulations: $\max S=0.93$ for $\gamma=1.075$ and $d t=0.10 \mathrm{UI}$.

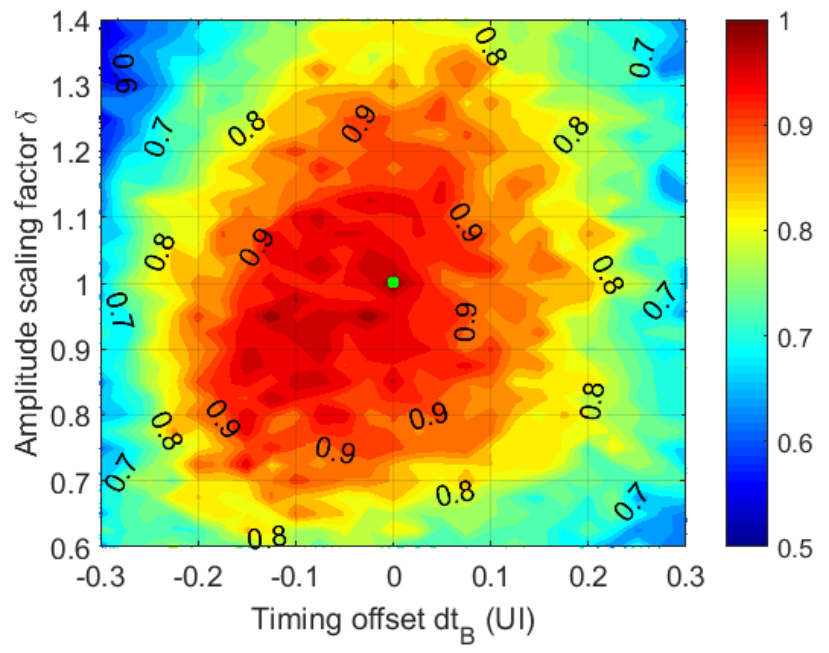

Fig. 9. Normalised eye diagram effective area $S$ after offline equalization as a function of the scaling factor $\delta$ and timing offset $d t_{B}$ of the $\widetilde{I_{B}}$ component for the Stretched A method for $16 \mathrm{~Gb} / \mathrm{s}$ transmission. The maximum value recorded $\max S=0.59$ for $\delta=1$ and $d t_{B}=0.0 \mathrm{UI}$ (green dot noted in plot).

\section{B. Sensitivity of $I_{A}^{\text {st }}$ to timing offsets}

In addition, an experimental study on the effect of the timing offset $t_{o f f}$ of the $I_{A}^{s t}$ component on the quality of the obtained waveforms is carried out. For a particular set of values for the scaling factor $\gamma$ and $d t$, a timing offset $t_{o f f}$ is introduced on the $I_{A}^{s t}$ component as illustrated in Fig. 15 and explained in section IV.B of Part I [1]. This offset, in contrast to the parameter $d t$ used in the previous section, represents the same type of time delay for both positive $(0 \rightarrow 1)$ and negative $(1 \rightarrow 0)$ transitions. Here, the quality of the generated optical waveform is tested for two sets of parameters $\gamma$ and $d t$ : $\gamma=1.08, d t=0.08 \mathrm{UI}$ and $\gamma=1.02, d t=0.10 \mathrm{UI}$. The modulating current waveform is calculated offline with the rate equation model for each offset and appropriately sampled. It is then generated with the AWG and applied onto the VCSEL. The output optical waveform is recorded and equalized with the 11-tap FFE offline. The effective area $S$ of the eye diagram after equalization is calculated and plotted in Fig. 10 as function of $t_{o f f}$. It can be clearly noticed that the introduction of the timing offset $t_{\text {off }}$ results in a fast degradation of the signal quality. The tolerance to the obtained $S \geq 0.5$ (normalized value of $\sim 0.85$ ) after equalization is found to be $\sim \pm 0.05$ UI only. A similar behavior was observed in the simulation studies reported in Part I (see Fig. 16) [1].

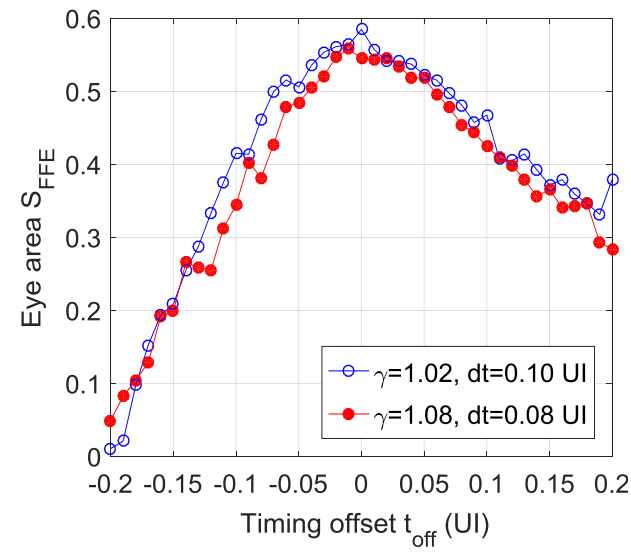

Fig. 10. Eye diagram effective area $S$ after equalization as a function of the timing offset $t_{\text {off }}$ for two sets of parameters for the $I_{A}^{S t}$ component of the Stretched A method and $16 \mathrm{~Gb} / \mathrm{s}$ data transmission.

As proposed in section IV.B in Part I and demonstrated via simulations [1], an adaptive gain scheme can be used to improve the tolerance of the Stretched A method to this type of timing offset. Here, the scheme is experimentally demonstrated for the two $d t$ values: $0.08 \mathrm{UI}$ and $0.10 \mathrm{UI}$. The optimum gains $a$ and $b$ of the $I_{A}^{s t}$ and $\widetilde{I_{B}}$ components are found with our laser equation model for each timing offset $t_{\text {off }}$ [Fig. 11(a)] and are used to produce the respective modulating current $\widetilde{I_{b c}}$. This is then generated with the AWG and applied onto the VCSEL. The output optical waveform is recorded for each offset $t_{\text {off }}$ and the effective area $S$ of the eye diagram after offline equalization is calculated. The results are shown in Fig. 11(b). The variation of the effective area $S$ when no gain adaptation is applied is also plotted in Fig. 11(b) to enable direct comparison. Similar behavior as that reported in the simulation section in Part I is observed (see Fig. 17 [1]) 
with much larger tolerances to timing offsets $t_{\text {off }}$ obtained. The tolerance to achieve $S \geq 0.5$ is increased to $\sim \pm 0.3$ UI with the use of the proposed adaptive scheme which is a 6 times improvement over when no adaption is applied. This is in good agreement with the respective value obtained in the simulation studies in Part I [1].

(a)

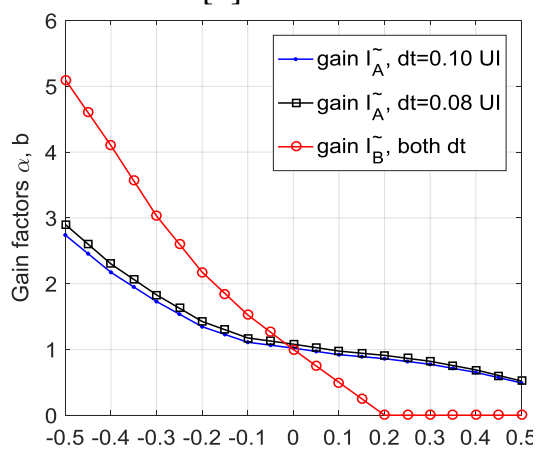

Timing offset $\mathrm{t}_{\text {off }}(\mathrm{UI})$

(b)

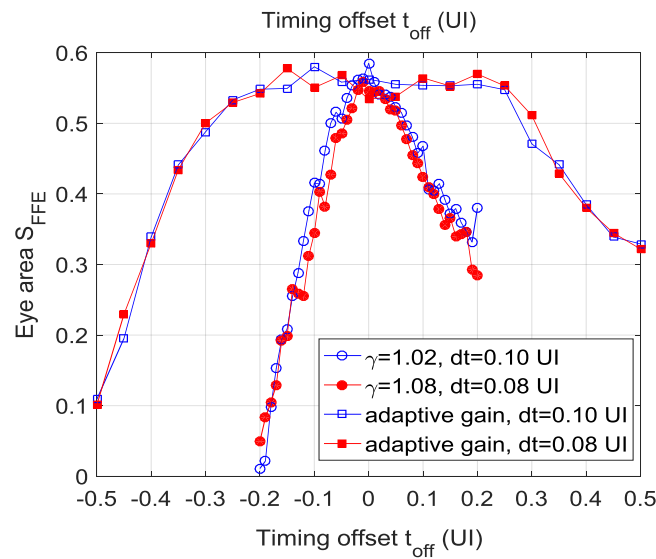

Fig. 11. (a) Gain factors $a$ and $b$ for the $I_{A}^{s t}$ and $\widetilde{I_{B}}$ components used in the adaptive gain scheme and (b) eye diagram effective area $S$ after offline equalization as a function of the timing offset $t_{\text {off } f}$.

\section{IMPLEMENTATION METHODS}

The simulation studies presented in Part I [1] and the experimental results reported here demonstrate that the Stretched A method has the potential to greatly improve the high data rate performance of DMLs. Different possible practical implementations of this method have been identified and are discussed in the following section.

\section{A. Prior work}

For various modulation formats, Karar et al. have reported the implementation of the back-calculation (ABCD) method with look-up tables [11] [5], digital signal processing [12] and digital-to-analog conversion [13, 14]. Over the past decade or so, other researchers have reported other methods for mitigating the non-linearity of a DML in short-reach optical links. These methods, briefly reviewed below, are however distinctively different to the back-calculation method explored by Karar et al. or the modified approximation proposed here.

The use of asymmetric pre-emphasis within the laser driver has been studied in [15-17]. Based on rate equation analysis, Raj et al. reported a non-linear equalizer which separately equalized the rising and falling edges with specifically tailored responses. The responses were achieved using different delays and amplitude scaling for a two-tap finite impulse response
(FIR) filter for each edge of the waveform [15]. The use of analog differentiation circuits to simplify a linear equalizer and reduce power dissipation by removing the need for inductors was reported in [18]. Kozlov et al. used an analog AC coupled FIR (effectively differentiators) to implement waveform shaping to mitigate the laser non-linearity [19]. Rather than use edge detection circuits to enhance the rising or falling edge, Yazaki et al., adjusted the DCD on the delay path, to obtain asymmetric pre-emphasis [17]. As far as we can determine, none of these methods created or claim to have created an approximation to the $I_{A}(t)$ component required by the back-calculation (ABCD) method. However, most probably created some form of modified $I_{B}(t)$ component to mitigate part of the laser non-linearity.

For PAM-4 modulation, Hecht et al., used a set of FIR filters, having $T$-spacing between the FIR taps, that could be selected based on the individual PAM-4 transition to be equalized [20]. In the field of long-distance communications, to mitigate the effects optical frequency chirp, Heismann et al. have reported the use of differentiation (first and higher orders) with analog circuits of the laser modulation current [21]. The laser was then modulated with linear combinations of the differentiated and initial modulation current. However, there was no attempt to "match" a desired optical output waveform per the back-calculation method.

\section{B. Potential implementations of the Stretched A method}

Potential implementations of the Stretched A method include the use of (i) analog circuits, (ii) digital circuits and appropriate filters and (iii) a T/2-spaced FIR filter to generate the total approximate modulating current $\widetilde{I_{b c}}(t)$. These methods are briefly explained below.

\section{1) Analog differentiation and/or integration circuits}

The linear current component $I_{C}(t)$ of the total modulating current can be directly calculated for the desired optical waveform $P(t)$. From inspection of eq. (2)-(5), the components $I_{A}^{s t}(t)$ and $\widetilde{I_{B}}(t)$ can be generated from $I_{C}(t)$ using a combination of adjustable differentiation, delay, summation and amplitude scaling circuits. In addition, a DCD circuit is required to generate the desired timing offset $d t$ of the $I_{A}^{s t}$ component. The three sub-currents $I_{A}^{s t}, \widetilde{I_{B}}$ and $I_{C}$ need to be aligned in time and added to the constant sub-current $I_{D}$ to produce the total modulation current $\widetilde{I_{b c}}$. Fig. 12 illustrates an example of the generation of the $\widetilde{I_{b c}}$ waveform using this type of implementation. 


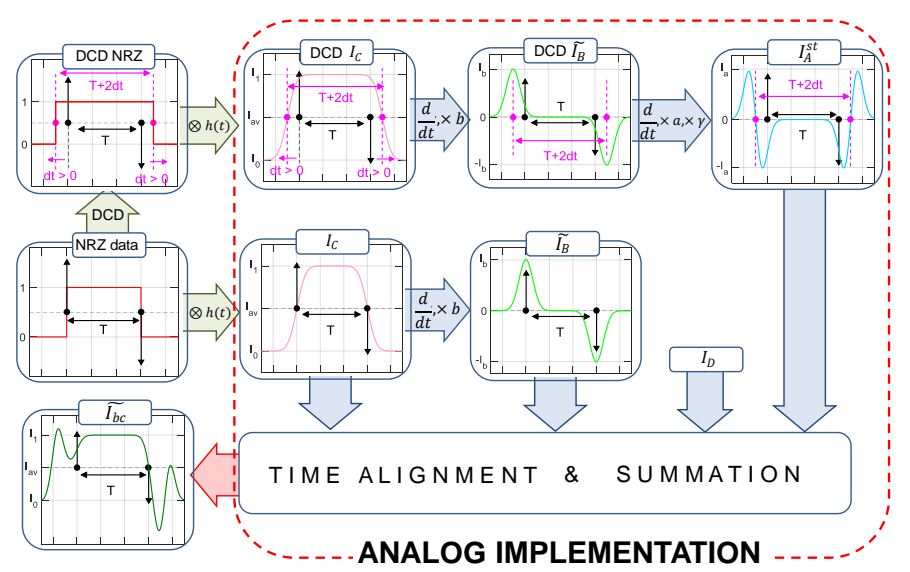

Fig. 12. Illustration of generation of the total modulating current $\widetilde{I_{b c}}(t)$ of the Stretched A method using analog electronics.

\section{2) Implementation via digital circuits and filtering}

The form of the $\widetilde{I_{A}}(t)$ and $\widetilde{I_{B}}(t)$ pulses suggest that the total modulation $\widetilde{I_{b c}}(t)$ of eq. (3) and (5) could be created based on the scaling, addition, synchronization and filtering of a dual di-code modulation format and the NRZ modulated signal followed by appropriate filtering. To implement the Stretched A method, circuits to add the DCD would also be required. The three sub-currents could be generated by using: (i) NRZ modulation as the basis for the $I_{c}(t)$ current, (ii) the edges of the NRZ waveform encoded as di-code NRZ pulses having the sign of the gradient of the edge as the basis for the $\widetilde{I_{B}}$ subcurrent (digital B pulses), (iii) the edges of the NRZ pulses after the DCD encoded as di-code bi-phase pulses having the sign of the edges as the basis for the $I_{A}^{s t}$ current (digital strA pulses).

The encoded waveforms could be formed by various methods. In Fig. 13 we schematically illustrate how these could be formed using digital circuits (such as FIR filters, delay lines) and basic analog circuitry (analog adders, analog gain stages, limiting amplifiers (LA), etc). It should be noted that the implementation depicted here assumes that, for simplicity, after forming the correctly scaled digital modulation waveform (digital $\widetilde{I_{b c}}$ pulses in Fig. 13), a single electrical filter is used to set the rise and fall times of the total modulation current $\widetilde{I_{b c}}(t)$. Theoretically, the best results would be obtained if each of the digital waves were separately (and appropriately) filtered before adding. However, it could be argued that using a single filter is attractive since it simplifies the implementation and uses fewer components.

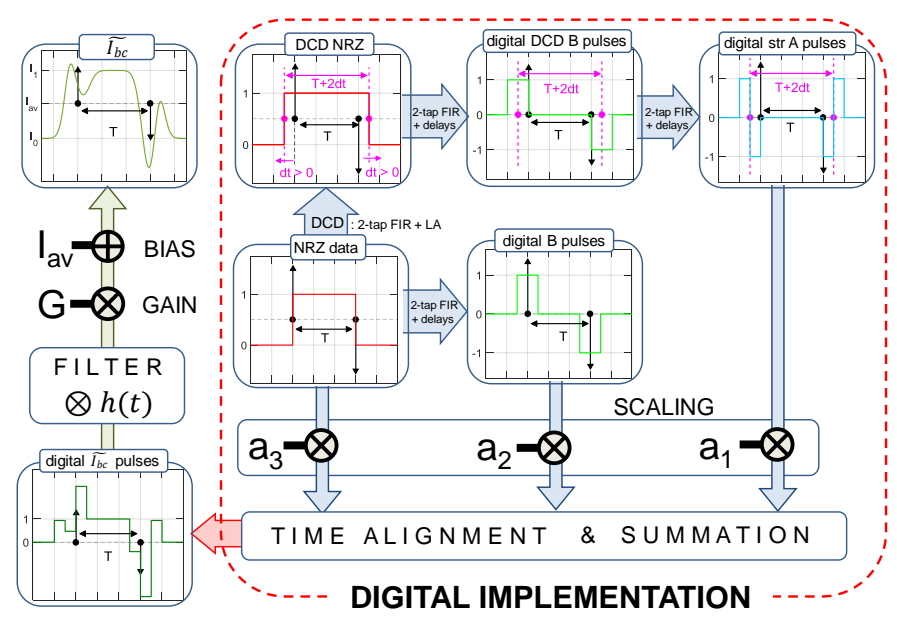

Fig. 13. Illustration of generation of the total modulating current $\widetilde{I_{b c}}(t)$ of the Stretched A method using digital circuits and filtering (FIR: finite impulse response filter, LA: limiting amplifier).

One potential advantage of this method would be that the signals could be created and synchronized using digital electronics before being scaled and combined with analog circuits. This implementation method might be particularly attractive as most of the functions could be implemented within a CMOS-based digital serializer-deserializer (SERDES) circuit. In addition, high data rate SERDES typically include linear equalizers to mitigate the linear distortion between their output and the next receiving chip or optical module. Therefore, the SERDES equalizer could be used to equalize the electrical path between the SERDES and a linear laser driver to help ensure the desired $\widetilde{I_{b c}}(t)$ is delivered to the laser.

\section{3) Implementation via a fractionally-spaced FIR filter}

Our studies have shown that an isolated NRZ ' 1 ' bit produces a specific back-calculated current pulse response $I_{b c}^{P R}(\mathrm{t})$ for the desired output optical waveform (Fig. 14). This current pulse response can be accurately approximated using a fractionally spaced FIR filter, with for example, $T / 2$ spacing between its taps [Fig. 14(b)]. Also, for simplicity and clarity, we have assumed the FIR filter was excited by an ideal return-to-zero (RZ) pulse of width $T / 2$.
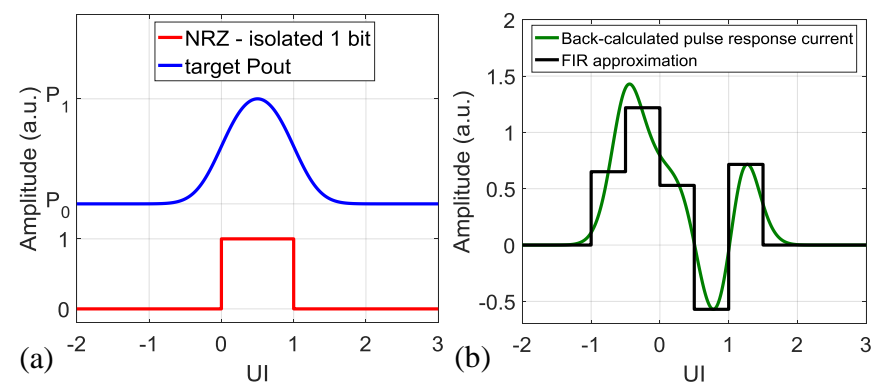

Fig. 14. Illustration of (a) the desired isolated optical pulse due to an isolated NRZ ' 1 ' bit and (b) the required back-calculated pulse response current and its FIR approximation using $T / 2$ spacing.

The approximate back-calculated modulation current $\widetilde{I_{b c}}(t)$, due to NRZ input data, may be generated using the FIR filter as follows. The tap weights of the FIR filter are set to approximate the $I_{b c}^{P R}$ pulse response of an isolated NRZ ' 1 ' bit 
when excited by an RZ ' 1 '. The input NRZ data is converted to RZ data which is then fed to the FIR filter. The filter output is appropriately scaled and a bias current is added to generate $\widetilde{I_{b c}}$. Fig. 15 illustrates this implementation. Furthermore, if an optical feedback method is available, the weights of the FIR filter could be adjusted based on the observed output optical waveform from the modulated VCSEL to ensure that the output optical waveform reproduces the target waveform as closely as possible.

The proposed method is distinctively different from the ones reported in the literature using asymmetric FFEs [15-17] as: (i) it is not based on the step responses of the edges of the optical waveform, (ii) produces a complete current pulse response and is equivalent to a pulse shaping filter (not an edge shaping filter per asymmetric FFEs), (iii) approximates the isolated pulse response of the back-calculated current and therefore includes an approximation of the $I_{A}$ sub-current. On the contrary, the asymmetric FFEs reported in literature essentially generate only some form of the $I_{B}$ component to mitigate part of the laser non-linearity.

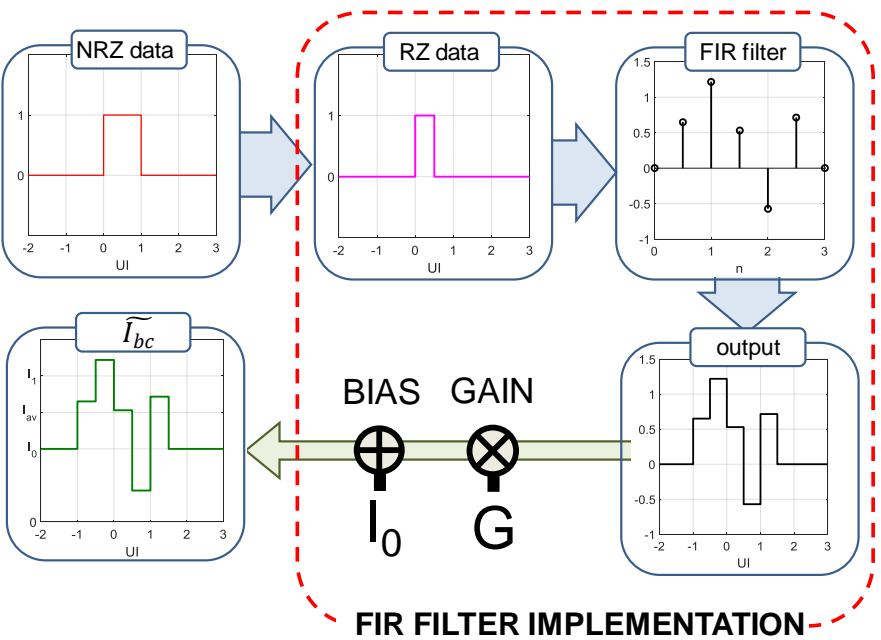

Fig. 15. Illustration of the generation of the approximate total modulating current $\widetilde{I_{b c}}(t)$ using a FIR filter with fractional spacing.

\section{CONCLUSIONS}

The first experimental demonstration of the application of the Stretched A method is reported using a $10 \mathrm{~Gb} / \mathrm{s} 850 \mathrm{~nm}$ multimode VCSEL. The method is used to generate high quality eye diagrams at $16 \mathrm{~Gb} / \mathrm{s}$ with greatly improved nonlinearity over conventional NRZ modulation. It is shown that the obtained eye diagrams exhibit very similar characteristics as those obtained using the back-calculated modulating current. In addition, an experimental sensitivity analysis on the generation of the required sub-currents is carried out demonstrating large tolerances. It is shown via experiments that a gain adaptive scheme can be used to improve the tolerance to timing offsets. Finally, potential hardware implementations of the proposed Stretched A method are presented.

\section{REFERENCES}

[1] N. Bamiedakis, D. G. Cunningham, and R. V. Penty, "Linearisation Method of DML-based Transmitters for Optical Communications, Part I:
Theory and Simulation Studies," IEEE Journal of Lightwave Technology, vol. submitted, 2021.

[2] G. M. Choudhury, J. Robert Lingle, J. Kamino, R. Shubochkin, D. Vaidya, D. Braganza, and Y. Sun, "Standards, Technology, Market and Industry Trends and Future Direction for MMF," in IWCS 2019 International Cable \& Connectivity Symposium. vol. paper 5.1, 2019, pp. $1-12$.

[3] A. Rodriguez-Perez, L. M. Torres, E. Prefasi, and R. P. d. Aranda, "Multi-Gigabit Transceivers for Optical Data Communications From the Standardization Perspective," IEEE Transactions on Circuits and Systems II: Express Briefs, vol. 68, pp. 56-62, 2021.

[4] D. G. Cunningham and I. H. White, "Advances in local area optical data communication systems," Reports on Progress in Physics, vol. 83, p. 075101, 2020.

[5] A. S. Karar, J. C. Cartledge, J. Harley, and K. Roberts, "Electronic PreCompensation for a 10.7-Gb/s System Employing a Directly Modulated Laser," Journal of Lightwave Technology, vol. 29, pp. 2069-2076, 2011.

[6] Ulm Photonics (Philips) 10 Gbps $850 \mathrm{~nm}$ VCSEL chips, part ULM85010-TN-N0101U.

[7] P. Westbergh, J. S. Gustavsson, B. Kogel, Ã. s. Haglund, and A. Larsson, "Impact of photon lifetime on high-speed VCSEL performance," IEEE Journal of Selected Topics in Quantum Electronics, vol. 17, pp. 1603$1613,2011$.

[8] S. W. Corzine, M. Mashanovitch, and L. A. Coldren, Diode lasers and photonic integrated circuits: Hoboken, New Jersey : Wiley, 2011.

[9] S. B. Healy, E. P. O. Reilly, J. S. Gustavsson, P. Westbergh, H. Ã..., A. Larsson, and A. Joel, "Active Region Design for High-Speed 850-nm VCSELs," IEEE Journal of Quantum Electronics, vol. 46, pp. 506-512, 2010.

[10]A. Larsson, "Advances in VCSELs for Communication and Sensing," IEEE Journal of Selected Topics in Quantum Electronics, vol. 17, pp. 1552-1567, 2011.

[11]A. S. Karar, M. YaÃ tez, Y. Jiang, J. C. Cartledge, J. Harley, and K. Roberts, "Electronic dispersion pre-compensation for $10.709-\mathrm{Gb} / \mathrm{s}$ using a look-up table and a directly modulated laser," Optics Express, vol. 19, pp. B81-B89, 2011.

[12]A. S. Karar, J. C. Cartledge, J. Harley, and K. Roberts, "Reducing the Complexity of Electronic Pre-compensation for the Nonlinear Distortions in a Directly Modulated Laser," in Advanced Photonics, Toronto, 2011, p. SPWA2.

[13]A. S. Karar, Y. Jiang, J. C. Cartledge, J. Harley, D. J. Krause, and K. Roberts, "Electronic precompensation of the nonlinear distortion in a 10 $\mathrm{Gb} / \mathrm{s}$ 4-ary ASK directly modulated laser," in 36th European Conference and Exhibition on Optical Communication (ECOC), 2010, pp. 1-3.

[14]A. S. Karar, "Electronic bandwidth enhancement of directly modulated semiconductor lasers," Optics Communications, vol. 439, pp. 137-140, 2019.

[15]M. Raj, M. Monge, and A. Emami, "A Modelling and Nonlinear Equalization Technique for a $20 \mathrm{~Gb} / \mathrm{s} 0.77 \mathrm{pJ} / \mathrm{b}$ VCSEL Transmitter in $32 \mathrm{~nm}$ SOI CMOS," IEEE Journal of Solid-State Circuits, vol. 51, pp. 1734-1743, 2016.

[16] K. Ohhata, H. Imamura, Y. Takeshita, K. Yamashita, H. Kanai, and N. Chujo, "Design of a 4 x $10 \mathrm{~Gb} / \mathrm{s}$ VCSEL Driver Using Asymmetric Emphasis Technique in 90-nm CMOS for Optical Interconnection," IEEE Transactions on Microwave Theory and Techniques, vol. 58, pp. 11071115, 2010.

[17]T. Yazaki, N. Chujo, H. Yamashita, T. Takemoto, Y. Lee, and Y. Matsuoka, "25-Gbps x 4 optical transmitter with adjustable asymmetric pre-emphasis in 65-nm CMOS," in IEEE International Symposium on Circuits and Systems (ISCAS), 2014, pp. 2692-2695.

[18]L. A. Valenzuela, H. Andrade, N. Hosseinzadeh, A. Maharry, C. L. Schow, and J. F. Buckwalter, "A 2.85 pJ/bit, 52-Gbps NRZ VCSEL Driver with Two-Tap Feedforward Equalization," in IEEE/MTT-S International Microwave Symposium (IMS), 2020, pp. 209-212.

[19] V. Kozlov and A. C. Carusone, "A 15Gb/s AC-Coupled VCSEL Driver with Waveform Shaping in 65nm CMOS," in IEEE Compound Semiconductor Integrated Circuit Symposium (CSICS), 2015, pp. 1-4.

[20]U. Hecht, N. Ledentsov, P. Scholz, M. Agustin, P. Schulz, N. N. Ledentsov, and F. Gerfers, "Non-Linear PAM-4 VCSEL Equalization and $22 \mathrm{~nm}$ SOI CMOS DAC for $112 \mathrm{Gbit} / \mathrm{s}$ Data Transmission," in 12th German Microwave Conference (GeMiC), 2019, pp. 115-118.

[21]F. L. Heismann, D. Mahgerefteh, and P. Tayebati, "Method and apparatus for generating signals with increased dispersion tolerance using a directly modulated laser transmitter," U. S. Patents, Ed., 2012. 